\title{
Fermionic Orbital Optimization in Tensor Network States
}

\author{
C. Krumnow, ${ }^{1}$ L. Veis, ${ }^{2,3}$ Ö. Legeza, ${ }^{2}$ and J. Eisert ${ }^{1}$ \\ ${ }^{1}$ Dahlem Center for Complex Quantum Systems, Freie Universität Berlin, 14195 Berlin, Germany \\ ${ }^{2}$ Strongly Correlated Systems "Lendület" Research Group, Wigner Research Centre for Physics, \\ Hungarian Academy of Sciences, 1525 Budapest, Hungary \\ ${ }^{3}$ J. Heyrovsky Institute of Physical Chemistry, Academy of Sciences of the Czech Republic, 18223 Prague, Czech Republic
}

(Received 22 May 2015; revised manuscript received 16 June 2016; published 18 November 2016)

\begin{abstract}
Tensor network states and specifically matrix-product states have proven to be a powerful tool for simulating ground states of strongly correlated spin models. Recently, they have also been applied to interacting fermionic problems, specifically in the context of quantum chemistry. A new freedom arising in such nonlocal fermionic systems is the choice of orbitals, it being far from clear what choice of fermionic orbitals to make. In this Letter, we propose a way to overcome this challenge. We suggest a method intertwining the optimization over matrix product states with suitable fermionic Gaussian mode transformations. The described algorithm generalizes basis changes in the spirit of the Hartree-Fock method to matrix-product states, and provides a black box tool for basis optimization in tensor network methods.
\end{abstract}

DOI: 10.1103/PhysRevLett.117.210402

Capturing strongly correlated quantum systems is one of the major challenges of modern theoretical and computational physics. Recent years have seen a surge of interest in the development of potent numerical methods based on tensor networks to approximate ground states of interacting lattice models [1-7], building upon the success of the density-matrix renormalization group (DMRG) [1]. It has become clear that such ideas are also applicable to fermionic systems [8-10], and even to systems of quantum chemistry [11-19], lacking the locality present in lattice models in condensed-matter systems. Such tools allow us in principle to approximate the full configuration interaction solution to good accuracy with reasonable effort, going, in instances, beyond conventional approaches to quantum chemistry, such as coupled cluster [20], configuration interaction, density-functional theory [21,22], or Monte Carlo based methods [23-25], as convincingly shown by first implementations of DMRG algorithms in quantum chemistry (QC-DMRG) [11-15]. Yet, there is a new obstacle to be overcome: Tensor network methods have originally been tailored to capture local interactions, and, consequently, ground states exhibiting short-range correlations and entanglement area laws [7]. Systems in quantum chemistry pose new challenges due to the inherent long-ranged interactions, which are present no matter in what basis the systems are expressed. New questions, hence, arise concerning the optimal topology and physical (orbital) basis used to construct the tensor network state [13-19,26-28].

In this Letter, we propose a novel approach towards making use of tensor network methods in quantum chemistry by suggesting an adaptive scheme of updating basis transformations "on the fly" in conjunction with tensor network updates. In this way, we bring together advantages of matrix product states-which can capture strongly correlated states, but are tailored to short-ranged correlations and low entanglement-and fermionic Gaussian mode transformations-for which entanglement is no obstacle, but non-Gaussian correlations are. We, hence, go significantly beyond previous approaches towards optimizing fermionic bases in tensor network approaches to quantum chemistry. Previous DMRG implementations in quantum chemistry allowing for an optimization of the physical basis restrict the mode transformations to permutations and separate the optimization over the basis and state such that multiple DMRG runs are necessary [29]. As a first attempt, basis optimizations using a few transformations have been implemented for tree tensor networks; however, this has been found to be unstable [19]. Mixing fermionic orbitals from an active space - the space considered here-with further ones from an additional external space has also been studied [30-32]. In these approaches orbital transformations are carried out again between different DMRG runs. In contrast to this, we perform the mode transformations within the active space in parallel to the state optimization and directly optimize the entanglement structure of the tensor network.

We focus on matrix-product states, but explain in what way the idea is generally applicable. We also discuss the role of symmetries and the geometry of the problem at hand. The basis optimization is incorporated into the standard two-site QC-DMRG and can be added to existing implementation without increasing the computational costs of the DMRG. The resulting scheme can be used in parallel to a ground state search or as a preprocessing step in which the physical basis is optimized in a first phase, restricting the bond dimension of the MPS used to medium values and calculating the final ground state in the optimized basis with higher accuracy.

System.- In this Letter, we are concerned with strongly correlated interacting fermionic models with a finite 
number of relevant modes as they appear in the quantum chemistry context. In the second quantized form the Hamitonian takes the form

$$
H=\sum_{i, j=1}^{n p} t_{i, j} c_{i}^{\dagger} c_{j}+\frac{1}{2} \sum_{i, j, k, l=1}^{n p} v_{i, j, k, l} c_{i}^{\dagger} c_{j}^{\dagger} c_{l} c_{k},
$$

where $c_{j}$ is a fermionic annihilation operator associated to the mode labeled $j$ satisfying the canonical anticommutation relations $\left\{c_{i}, c_{j}\right\}=0$ and $\left\{c_{i}^{\dagger}, c_{j}\right\}=\delta_{i, j}$, and the coupling $t$ and $v$ are such that $H$ is Hermitian. $p$ denotes the number of different fermion species present for each of the $n$ orbitals, e.g., spin-up and -down electrons. The one particle modes form the basis of single particle Hilbert space $\mathcal{H}_{n p}$. Any fermionic state will be an element of the fermionic Fock space $\mathcal{F}=\bigoplus_{k=0}^{n p} \wedge^{k} \mathcal{H}_{n p}$, where $\wedge$ denotes the exterior product and $\wedge^{0} \mathcal{H}_{n p}=\mathbb{C}$, with a basis formed of all Slater determinants $|x\rangle$, where $x \in\{0,1\}^{n p}$, of the initial single particle modes. We refer to this basis as the physical basis. The Jordan Wigner transformation establishes an isomorphism between $\mathcal{F}$ and the Hilbert space of $n$ qudits $\mathcal{H}_{d}^{\otimes n}=\mathbb{C}^{d^{n}}$ with $p=\log _{2} d$. By choosing any ordering of the orbitals such systems can be viewed as onedimensional lattices of $n$ sites with long-range interactions.

MPS and general idea.-For a one-dimensional quantum lattice with $n$ sites, where each site is described by a $d$-dimensional Hilbert space $\mathcal{H}_{d}$, an open boundary matrix product state (MPS) vector takes the general form

$$
|\psi\rangle=\sum_{\alpha_{1}, \ldots, \alpha_{n}=1}^{d} A_{[1]}^{\alpha_{1}} \ldots A_{[n]}^{\alpha_{n}}\left|\alpha_{1}\right\rangle \otimes \ldots \otimes\left|\alpha_{n}\right\rangle,
$$

where $A_{[m]}^{\alpha_{m}} \in \mathbb{C}^{D_{m-1} \times D_{m}}$ and $\{|\alpha\rangle\}$ form a basis of $\mathcal{H}^{d}$ and $D_{0}=1=D_{n}$. If the bond dimension $D \in \mathbb{N}$ is allowed to vary arbitrarily over different sites, every quantum state of the lattice can be written as in Eq. (2) [33]. Restricting the maximal bond dimension along the chain to a fixed value $D_{\text {max }}$ creates the submanifold $\mathcal{M}_{D_{\max }}$ of the full state space. Approximations of the ground state of a given Hamiltonian within this submanifold can be found using the density matrix renormalization group algorithm (DMRG) which, as an alternating least square method, optimizes the entries of the MPS tensors $\left(A_{[m]}\right)_{m}$ iteratively [28,34-36].

The freedom one has in this construction is a redefinition of the fermionic modes by a linear transformation. Linear transformations of a set of fermionic annihilation operators $\left\{c_{i}\right\}$ to a new set $\left\{d_{i}\right\}$ satisfying the canonical anticommutation relations are captured by $c_{i}=\sum_{j=1}^{n p} U_{i, j} d_{j}$, with a unitary mode transformation $U \in U(n p)$. This change of the single particle modes induces a transformation of the physical basis of $\mathcal{F}$. Under this change of basis a fermionic state vector $|\psi(\mathbb{1})\rangle$ transforms to $|\psi(U)\rangle=G(U)|\psi(\mathbb{1})\rangle$ with the Gaussian unitary transformation $G(U)=$ $\exp \left[\sum_{i, j}\left(\ln U^{\dagger}\right)_{i, j} c_{i}^{\dagger} c_{j}\right]$ acting in Fock space. The transformation on $\mathbb{C}^{d^{n}}$ induced from the Jordan Wigner transformation is given by $g(U)=\bigoplus_{k=0}^{n p} \wedge^{k} U^{\dagger}$, where $\bigwedge^{0} U^{\dagger}=1$.

We now turn to describing ground states of fermionic Hamiltonians with MPS expressed in a given basis, where the approximatability of the states strongly depends on the choice of basis [26,27]. Specifically, denoting the Hamiltonian written in terms of the transformed modes by $H(U)=G(U)^{\dagger} H G(U)$, we are interested in the solutions of

$$
\left(U_{\mathrm{opt}},\left|\psi_{\mathrm{opt}}\right\rangle\right)=\underset{U \in U(n p),|\psi\rangle \in \mathcal{M}_{D_{\max }}}{\operatorname{argmin}}\langle\psi|H(U)| \psi\rangle .
$$

Note that the Hartree-Fock method is readily included in Eq. (3) by the case $D_{\max }=1$, when $|\psi\rangle$ is restricted to be a Slater determinant. Identifying the optimal or close-to optimal basis for a general Hamiltonian and $D_{\max }$ in the sense of Eq. (3) would provide a deeper understanding of the entanglement structure of ground states appearing in quantum chemistry, but since this is a nonconvex problem, approximate solutions are accessible only. Here, we take an approach that iteratively finds close to optimal solutions numerically by optimizing over the ansatz-class depicted in Fig. 1.

Compositions of local mode transformations.-In order to calculate approximations to the solutions of Eq. (3) and avoiding stability and performance issues of a direct global optimization, we perform successive local mode transformations in parallel to a two-site QC-DMRG and use a few additional global reorderings of the orbitals as in Refs. [27,29] to leave local minima during the optimization process. Given a state vector $|\psi\rangle$, a site-index $m \in[n-1]$ and a cost function $f_{m}$ which will be discussed below we solve

$$
U_{\mathrm{opt}}^{\mathrm{loc}}=\underset{U \in V}{\arg \min } f_{m}\left(\left|\psi\left(\mathbb{1}_{p m} \oplus U \oplus \mathbb{1}_{p n-p m-2 p}\right)\right\rangle\right),
$$

with $\mathbb{1}_{k}$ denoting the $k$-dimensional identity matrix and $V \subset$ $U(2 p)$ needs to be chosen depending on the symmetries of the system. The global basis change is then composed of

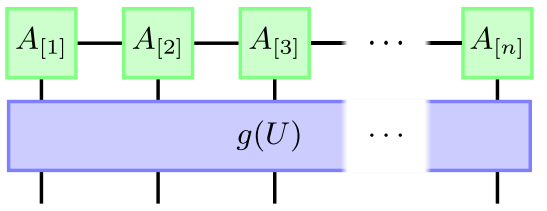

FIG. 1. Illustration of the general ansatz class of an MPS with varying physical basis, where $g(U)$ is a Gaussian transformation defined by a mode transformation $U \in U(n p)$ as described in the main text. 
local unitaries which are solutions of Eq. (4) for different $m$ and act nontrivially on overlapping areas of the lattice and intermediate global reorderings of the lattice sites.

The cost function is chosen according to the following paradigm. The bond dimension needed for a bipartition of the system to approximate a state up to a predefined accuracy can be upper bounded using the Rényi entropies $S^{\alpha}\left(\rho_{\text {red }}\right)$ of the reduced state for $\alpha<1$ [37], where $S^{\alpha}(\rho)=\log \operatorname{tr} \rho^{\alpha} /(1-\alpha)$. We, therefore, iteratively minimize the $S^{\frac{1}{2}}$ entropy over the chosen bipartition by using the cost function $f_{m}^{(1)}(|\psi\rangle)=\left\|\Sigma_{\psi}^{m}\right\|_{1}$, where $\Sigma_{\psi}^{m}$ denotes the Schmidt spectrum of $|\psi\rangle$ for a bipartiting cut between sites $m$ and $m+1$. With increasing dimension of $V$, which growth with the number of species per orbital $p$, and bond dimension $D_{\max }$ the optimization of $f_{m}^{(1)}$ becomes slow. Efficiency can be gained by minimizing $f_{m}^{(4)}=-\left\|\Sigma_{\psi}^{m}\right\|_{4}^{4}$ of which we can calculate the gradient $\nabla_{U_{i, j}} f_{m}^{(4)}\left(\left|\psi\left(\mathbb{1}_{p m} \oplus U \oplus \mathbb{1}_{p n-p m-2 p}\right)\right\rangle\right)$ analytically and efficiently in the bond dimension as shown in the Supplemental Material [38]. The optimization of $S^{2}$ will not lead to certified bounds on the required bond dimension, but will favor stronger decays in the Schmidt spectrum similar as the minimization of $S^{\frac{1}{2}}$.

The results presented here have been obtained by optimizing the one norm of the Schmidt spectrum, $f_{m}^{(1)}$. The optimization of $f_{m}^{(4)}$ can be applied if $V$ has a higher dimension, which appear for $p>1$ if the system lacks symmetries. Both the choice of the cost function and symmetries influence the choice of $V$ as argued in the following.

Optimization set.-In the presence of symmetries, choosing a physical basis which can be labeled by good quantum numbers decouples different symmetry sectors in the coefficient tensors of the Hamitonian and the MPS and allows for more efficient computations. Only mode transformations, which commute with the generators of the symmetry transformations, will preserve the structure imposed by the symmetry.

In general, QC-DMRG algorithms only exploit a subgroup of the full symmetry group of a specific Hamiltonian, such as conservation of the number of particles, spin reflection symmetries, Abelian point group symmetries, or a $S U(2)$ spin rotation symmetry [1,16,39-42] (see also Ref. [28]). Here, we consider for the states the case of particle number conservation of each species, which is an Abelian symmetry and allows for an easy implementation of symmetric MPS [43] and want the local mode transformations to respect the $S U(2)$ symmetry of the considered systems. The admissible transformations in this case are of the form $U=U_{n}^{\oplus p}$ with $U_{n} \in U(n)$ acting on one species of fermions.

The cost functions $f_{m}$ chosen above depend only upon the Schmidt spectrum of the state for a cut between sites $m$ and $m+1$ and are therefore insensitive to mode transformations of the form $U_{m} \oplus U_{m+1}$ with $U_{q} \in U(p)$ acting only on the modes associated to the lattice site $q$. To obtain a nonredundant parametrization of the unitaries used in the optimization in Eq. (4) we restrict it to the set of left cosets $U(2 p) / U(p) \times U(p)$, which is isomorphic to the Grassmann manifold $G(2 p, p)$. Efficient implementations of optimization algorithms such as the conjugate gradient method within Grassmann manifolds using $2 p^{2}$ parameters are described in Refs. [44,45]. If we restrict ourselves to mode transformations that preserve the $S U(2)$ symmetry, the relevant mode transformations are parametrized by $G(2,1)$, leaving 2 optimization parameters in each step. Focusing on this case here with medium values for $D_{\max }$, we can obtain $U_{\mathrm{opt}}^{\mathrm{loc}}$ of $f_{m}^{(1)}$ by using gradient free schemes such as the Nelder-Mead method, due to the small number of parameters.

Algorithm.-Combined with an approximation of the ground state of a given Hamiltonian, local mode transformations naturally extend a two-site DMRG. A single two-site DMRG step results in a blocked tensor $A_{[m, m+1]} \in \mathbb{C}^{d^{2} \times D_{m-1} \times D_{m+1}}$. In the generic case, restoring the MPS format in Eq. (2) by decomposing the blocked tensor into local tensors $A_{[m]}^{\prime} \in \mathbb{C}^{d \times D_{m-1} \times D_{m}^{\prime}}$ and $A_{[m+1]}^{\prime} \in$ $\mathbb{C}^{d \times D_{m}^{\prime} \times D_{m+1}}$ will lead to $D_{m}^{\prime}>D_{\max }$ such that the found state needs to be projected into $\mathcal{M}_{D_{\max }}$ by discarding the $D_{m}^{\prime}-D_{\max }$ smallest values of the resulting Schmidt spectrum $\sum_{\psi}^{m}$. The projection yields a truncation error $\epsilon_{t}=\sum_{i} \sigma_{i}^{2}$, where $\sigma_{i} \in \sum_{\psi}^{m}$ are the discarded singular values. If we allow for a local mode transformation before the blocked tensor is decomposed, the truncation error can be reduced.

Using the gauge invariance of MPS, we bring the MPS to a mixed normalized state; i.e., matrices of sites $q<m$ are left normalized whereas matrices associated to sites $q>$ $m+1$ are right normalized $[2,46]$. We can then calculate $\Sigma_{\psi}^{m}$ from the blocked tensor $A_{[m, m+1]}$. We optimize the basis by solving Eq. (4) while keeping expectation values of the state, such as the energy, constant by using $\langle\psi|H| \psi\rangle=$ $\left\langle\psi(U)\left|H\left(U^{\dagger}\right)\right| \psi(U)\right\rangle$ with $U=\mathbb{1}_{p m} \oplus U_{\mathrm{opt}}^{\mathrm{loc}} \oplus \mathbb{1}_{p n-p m-2 p}$. As the mode transformation acts nontrivially only on sites $m, m+1$ the transformed state vector $|\psi\rangle$ can be represented by

$$
\begin{aligned}
& A_{[k]}(U)=A_{[k]}(\mathbb{1}), \quad k \in[n] \backslash\{m, m+1\}, \\
& A_{[m, m+1]}^{\alpha, \beta}(U)=\sum_{\alpha^{\prime}, \beta^{\prime}} g(U)_{(\alpha, \beta),\left(\alpha^{\prime}, \beta^{\prime}\right)} A_{[m, m+1]}^{\alpha^{\prime}, \beta^{\prime}}(\mathbb{1}) .
\end{aligned}
$$

Operators such as the Hamiltonian can be transformed efficiently using their second quantized representation. For an operator $O=\sum_{i_{1}, \ldots, i_{s}, j_{1}, \ldots, j_{r}=1}^{n p} o_{i_{1}, \ldots, i_{s}, j_{1}, \ldots, j_{r}} c_{i_{1}}^{\dagger} \ldots c_{i_{s}}^{\dagger} c_{j_{r}} \ldots c_{j_{1}}$ with $o=o(\mathbb{1})$, the coefficients transform under a mode 
transformation according to $o(U)=\left(U^{\dagger}\right)^{\otimes s} o(\mathbb{1}) U^{\otimes r}$. As most operators of interest, e.g., the Hamiltonian, contain terms with small $s$ and $r$, those transformations can be implemented efficiently; with cost scaling as $O\left((n p)^{s+r-1}\right)$ for local transformations.

Standard QC-DMRG algorithms use complementary operators $[12,14,15]$ in order to reduce the computational cost of each DMRG step. In the Appendix we show that complementary operators transform as general operators under local mode transformations and argue that local mode transformation can be found and applied in a time not exceeding the computational cost of a single DMRG step. This allows us to keep the structure and the computational complexity of the two-site DMRG algorithm and perform basis optimizations essentially for free with the algorithm in Table I.

Numerical results.-We use a QC-DMRG algorithm which uses the dynamical block state selection approach [15] and configuration interaction based dynamically extended active space [16] procedure to accelerate the convergence and adapt the basis of the physical space by the algorithm described above. As a test system, we have chosen the electron configuration of a beryllium ring built from $6 \mathrm{Be}$ atoms. This system has recently been investigated [27] and a strong dependence of the convergence of the DMRG from the initial basis was observed. We investigate the molecule in a stretched geometry with an interatomic distance of $3.3 \AA$. As initial bases we use the Hartree-Fock (HF) basis of the system and a localized basis derived from the HF orbitals by a Foster-Boys localization [47]. Such localized orbitals are widely used in QC-DMRG calculations and are known to yield a better convergence for the Be ring [27].

Starting from the according initial basis we iteratively apply the following scheme: we run the standard QC-DMRG for 2 sweeps, perform 8 additional sweeps together with the local mode transformation as described in Table I, and reorder the basis according to its mutual information patterns [29]. Hereby, we either fix the truncation error $\epsilon_{\text {trc }}$ made in each step or set a hard cut on $D_{\max }$. The results of our calculations are show in Fig. 2. In the left panel of Fig. 2 we show how the bond dimension behaves for a ground state search with a bounded truncation

TABLE I. Two-site DMRG with adaptive mode transformations.

$1 \quad$ iterate over neighboring sites $m \in[n-1]$ :

2 get blocked tensor $A_{[m, m+1]}^{\alpha, \beta}$ (e.g., from two-site DMRG)

3 calculate (local) minimum $U_{\mathrm{opt}}^{\mathrm{loc}}$ of $f_{m}(|\psi(\mathbb{1} \oplus U \oplus \mathbb{1})\rangle)$

$4 \quad$ if $f_{m}\left|\psi\left(\mathbb{1} \oplus U_{\mathrm{opt}}^{\text {loc }} \oplus \mathbb{1}\right)\right\rangle<f_{m}(|\psi(\mathbb{1})\rangle)$ :

5 transform $|\psi\rangle$ with $U$ by updating

$$
\begin{aligned}
& A_{[m, m+1]}^{\alpha, \beta}=\sum_{\alpha^{\prime}, \beta^{\prime}=1}^{d} g\left(U_{\mathrm{opt}}^{\mathrm{loc}}\right)_{(\alpha, \beta),\left(\alpha^{\prime}, \beta^{\prime}\right)} A_{[m, m+1]}^{\alpha^{\prime}, \beta^{\prime}} \\
& \text { and transform relevant operators with } U^{\ddagger}
\end{aligned}
$$

6

calculate $A_{[m]}, A_{[m+1]}$ by decomposing $A_{[m, m+1]}$

with truncation and update MPS with new tensors error $\epsilon_{\text {trc }} \leq 10^{-6}$ and $D_{\min }=64$ for a calculation in the HF basis and optimized bases obtained by the above scheme starting from the HF orbitals.

It is key to the method proposed that the optimization of the basis leads to a significant decrease of needed resources already in the first iteration, where after the tenth iterations of basis optimization the needed bond dimension is more than 1 order of magnitude smaller than in the unoptimized orbitals. For realistic applications of the scheme, intermediate high bond dimensions during the calculation need to be avoided. The right panel of Fig. 2 shows the relative error in energy reached when performing a calculation with $D_{\max }=256$ starting in the HF and localized basis. As noted before, the localized basis allows for a more efficient approximation of the ground state than the HF basis. The basis optimization allows us to further significantly optimize both the HF and the localized basis. Starting from the HF orbitals we obtain a basis for which the relative error in energy drops by 1 order of magnitude from $1.52 \times 10^{-4}$ to $1.2 \times 10^{-5}$. Beginning at the localized basis allows us to reduce the relative error in energy from $3.7 \times 10^{-6}$ to $8.3 \times 10^{-7}$. Note that the energy in the optimized basis
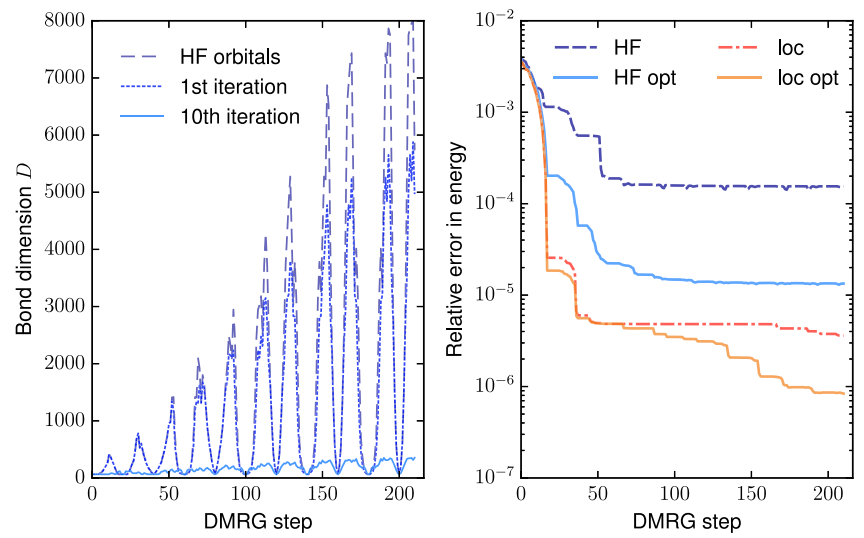

FIG. 2. Numerical results for the Be ring of 6 Be atoms with interatomic distance of $3.3 \AA$. All calculations have been performed with a $U(1) \times U(1)$ symmetric open boundary MPS and local mode transformations which keep the $S U(2)$ symmetry of the Hamiltonian. Both diagrams show results of the described optimization for the physical basis. In the left panel we show the bond dimension needed for a bounded truncation error $\epsilon_{\text {trc }} \leq 10^{-6}$ and $D_{\min }=64$ when starting in the HF basis. The dark blue dashed line corresponds to a calculation in the HF basis, the blue dotted and light blue line correspond to the first and the 10th iteration of the calculation with basis optimization. The right panel compares the relative error in energy $\left(\langle\psi|H| \psi\rangle-E_{0}\right) / E_{0}$ obtained by calculations with $D_{\max }=256$, where the reference value for the ground state energy $E_{0}$ has been obtained from a calculation with $D_{\max }=2048$ in the localized basis. The dark blue dashed and red dashed-dotted lines show the results for a calculation in the HF and localized basis, respectively. In light blue and orange we plot the relative error of the 15th and 10th iteration of the calculation with basis optimization starting in the $\mathrm{HF}$ and localized basis, respectively. 
starting from the HF orbitals is slightly worse than the energy obtained in the localized orbitals, reflecting the fact that finding the optimal basis is a hard global optimization problem.

We have repeated similar calculations for different configurations of the Be ring; at the equilibrium configuration and close to the avoided crossing. In each case we have been able to find a physical basis allowing for a more efficient approximation of the ground state. This illustrates that the above scheme can significantly and efficiently optimize a given initial basis. As the local mode transformation can be added with no increase of the computational cost to an existing two-site DMRG and typically yield already in the first iteration of the basis optimization a significant improvement of the basis our scheme extends the standard QC two-site DMRG.

Conclusion and perspectives. - In this work, we have presented a scheme that adapts the physical basis an MPS is formulated in by applying Gaussian transformations in an approach that is truly variational and controlled in the error. Incorporating local Gaussian transformations into the twosite DMRG algorithm allows us to optimize both the basis and the MPS iteratively. The resulting algorithm successfully optimizes the physical basis such that distinctly better approximations of the ground state by an MPS can be identified.

It should be manifest from the description of the method that the same idea is equally applicable to other tensor networks, due to the locality of the transformations. In particular, tree-tensor network approaches $[19,48,49]$ can readily be combined with the methods laid out here. Similarly, they are expected to be helpful for periodic boundary condition MPS [36,50] and 2D lattice systems $[3,4,35]$. In addition, the above scheme can be directly combined with recent developments for the time evolution of MPS [51] in order to obtain a time evolution with variational physical basis.

Our general strategy-of combining tensor networks with fermionic transformations-complements the recent interesting approach of Ref. [52], which is similar in mind set, but where these two components are put together in the opposite order. There, a matrix-product operator is applied onto a free fermionic wave function. In contrast to that approach, we here retain efficient contractibility, however. The approach taken in this work can also be seen as a variational principle that allows us to find the optimal fermionic tensor network in Ref. [53], where a fixed fermionic basis change is being made use of. Widening the scope, these tools seem also helpful in related approaches making use of a big data machinery to capture strongly correlated quantum systems. For example, compressed sensing ideas can help finding localized Wannier functions [54,55], which in turn can be made use of in density functional theory $[21,22]$. The approach pursued here may also help in identifying the deterministic space in semistochastic full configuration interaction quantum Monte Carlo calculations [23,25], which complements other works combining tensor network approaches with quantum Monte Carlo methods [56,57]. In conjunction with the tools developed here, a combined approach close to optimally representing fermionic correlated states seems within reach.

We would like to thank G. Chan, E. Fertitta, V. Murg, A. Nagy, V. Nebendahl, J. Rodriguez-Laguna, R. Schneider, T. Szilvási, and F. Verstraete for discussions. J.E. and C. K. acknowledge support from the EU (SIQS, RAQUEL, AQUS), the ERC (TAQ), the DFG (EI 519/7-1, CRC 183), the Templeton Foundation, and the Studienstiftung des Deutschen Volkes, L. V. and Ö. L. from the Czech Science Foundation, Grant No. 16-12052S and the Hungarian Research Fund (OTKA), Grants No. NN110360 and No. K120569.

[1] S. R. White, Phys. Rev. Lett. 69, 2863 (1992).

[2] U. Schollwöck, Ann. Phys. (Amsterdam) 326, 96 (2011).

[3] F. Verstraete, V. Murg, and J. Cirac, Adv. Phys. 57, 143 (2008).

[4] R. Orús, Ann. Phys. (Berlin) 349, 117 (2014).

[5] N. Schuch, in Quantum Information Processing, Lecture notes for the 44th IFF Spring School in Jülich (2013), Jülich.

[6] J. Eisert, Model. Simul. 3, 520 (2013).

[7] J. Eisert, M. Cramer, and M. B. Plenio, Rev. Mod. Phys. 82, 277 (2010).

[8] T. Barthel, C. Pineda, and J. Eisert, Phys. Rev. A 80, 042333 (2009).

[9] P. Corboz, G. Evenbly, F. Verstraete, and G. Vidal, Phys. Rev. A 81, 010303 (2010).

[10] C. V. Kraus, N. Schuch, F. Verstraete, and J. I. Cirac, Phys. Rev. A 81, 052338 (2010).

[11] G. Fano, F. Ortolani, and L. Ziosi, J. Chem. Phys. 108, 9246 (1998).

[12] S. R. White and R. L. Martin, J. Chem. Phys. 110, 4127 (1999).

[13] A. O. Mitrushenkov, G. Fano, F. Ortolani, R. Linguerri, and P. Palmieri, J. Chem. Phys. 115, 6815 (2001).

[14] G. K.-L. Chan and M. Head-Gordon, J. Chem. Phys. 116, 4462 (2002).

[15] O. Legeza, J. Röder, and B. A. Hess, Phys. Rev. B 67, 125114 (2003)

[16] O. Legeza and J. Sólyom, Phys. Rev. B 68, 195116 (2003).

[17] G. Moritz, B. A. Hess, and M. Reiher, J. Chem. Phys. 122, 024107 (2005).

[18] G. K.-L. Chan and S. Sharma, Annu. Rev. Phys. Chem. 62, 465 (2011).

[19] V. Murg, F. Verstraete, O. Legeza, and R. M. Noack, Phys. Rev. B 82, 205105 (2010).

[20] I. Shavitt and R. J. Bartlett, Many-Body Methods in Chemistry and Physics: MBPT and Coupled-Cluster Theory (Cambridge University Press, Cambridge, 2009).

[21] W. Kohn and L. J. Sham, Phys. Rev. 140, A1133 (1965). 
[22] M. Fuchs and M. Scheffler, Comput. Phys. Commun. 119, 67 (1999).

[23] N. S. Blunt, S. D. Smart, J. A. F. Kersten, J. S. Spencer, G. H. Booth, and A. Alavi, J. Chem. Phys. 142, 184107 (2015).

[24] D. Ceperley, G. V. Chester, and M. H. Kalos, Phys. Rev. B 16, 3081 (1977).

[25] F. R. Petruzielo, A. A. Holmes, H. J. Changlani, M. P. Nightingale, and C. J. Umrigar, Phys. Rev. Lett. 109, 230201 (2012).

[26] J. Rissler, R. M. Noack, and S. R. White, Chem. Phys. 323, 519 (2006).

[27] E. Fertitta, B. Paulus, G. Barcza, and O. Legeza, Phys. Rev. B 90, 245129 (2014).

[28] S. Szalay, M. Pfeffer, V. Murg, G. Barcza, F. Verstraete, R. Schneider, and Ö. Legeza, Int. J. Quantum Chem. 115, 1342 (2015).

[29] G. Barcza, O. Legeza, K. H. Marti, and M. Reiher, Phys. Rev. A 83, 012508 (2011).

[30] D. Zgid and M. Nooijen, J. Chem. Phys. 128, 144116 (2008).

[31] D. Ghosh, J. J. Hachmann, T. Yanai, and G. K. L. Chan, J. Chem. Phys. 128, 144117 (2008).

[32] H.-G. Luo, M.-P. Qin, and T. Xiang, Phys. Rev. B 81, 235129 (2010).

[33] G. Vidal, Phys. Rev. Lett. 91, 147902 (2003).

[34] S. Östlund and S. Rommer, Phys. Rev. Lett. 75, 3537 (1995).

[35] F. Verstraete and J. I. Cirac, arXiv:cond-mat/0407066.

[36] F. Verstraete, D. Porras, and J. I. Cirac, Phys. Rev. Lett. 93, 227205 (2004).

[37] F. Verstraete and J. I. Cirac, Phys. Rev. B 73, 094423 (2006).

[38] See Supplemental Material at http://link.aps.org/ supplemental/10.1103/PhysRevLett.117.210402 for details of the arguments presented in the main text.
[39] I. P. McCulloch, J. Stat. Mech. (2007) P10014.

[40] A. I. Tóth, C. P. Moca, O. Legeza, and G. Zaránd, Phys. Rev. B 78, 245109 (2008).

[41] S. Sharma and G. K.-L. Chan, J. Chem. Phys. 136, 124121 (2012).

[42] S. Wouters, P. A. Limacher, D. Van Neck, and P. W. Ayers, J. Chem. Phys. 136, 134110 (2012).

[43] S. Singh, R. N. C. Pfeifer, and G. Vidal, Phys. Rev. B 83, 115125 (2011).

[44] A. Edelman, T. Arias, and S. Smith, SIAM J. Matrix Anal. Appl. 20, 303 (1998).

[45] J. Manton, IEEE Trans. Signal Process. 50, 635 (2002).

[46] D. Perez-Garcia, F. Verstraete, M. M. Wolf, and J. I. Cirac, Quantum Inf. Comput. 7, 401 (2007).

[47] S. F. Boys, Rev. Mod. Phys. 32, 296 (1960).

[48] N. Nakatani and G. K.-L. Chan, J. Chem. Phys. 138, 134113 (2013).

[49] V. Murg, F. Verstraete, R. Schneider, P. R. Nagy, and Ö. Legeza, J. Chem. Theory Comput. 11, 1027 (2015).

[50] P. Pippan, S. R. White, and H. G. Evertz, Phys. Rev. B 81, 081103(R) (2010).

[51] J. Haegeman, C. Lubich, I. Oseledets, B. Vandereycken, and F. Verstraete, Phys. Rev. B 94, 165116 (2016).

[52] C.-P. Chou, F. Pollmann, and T.-K. Lee, Phys. Rev. B 86, 041105(R) (2012).

[53] A. J. Ferris, Phys. Rev. Lett. 113, 010401 (2014).

[54] V. Ozolins, R. Lai, R. Caflisch, and S. Osher, Proc. Natl. Acad. Sci. U.S.A. 110, 18368 (2013).

[55] J. C. Budich, J. Eisert, E. J. Bergholtz, S. Diehl, and P. Zoller, Phys. Rev. B 90, 115110 (2014).

[56] S. Wouters, B. Verstichel, D. Van Neck, and G. K.-L. Chan, Phys. Rev. B 90, 045104 (2014).

[57] B. K. Clark and H. J. Changlani, arXiv:1404.2296. 